\title{
Predição do escoamento superficial e consequentes alagamentos em centros urbanos
}

Os alagamentos são um problema em vários centros urbanos decorrentes da descaracterização natural do solo e do aumentando do escoamento superficial. A caracterização hidrodinâmica dos solos pode ser realizada por ensaios de campo ou laboratoriais visando a predição destes alagamentos. Sendo assim, objetivouse estimar o escoamento superficial de água no solo por meio de simulações utilizando o Hydrus-1D, para explicar os alagamentos que ocorrem numa área urbana. Para obtenção dos parâmetros hidrodinâmicos seguiu-se a metodologia do Beerkan of Estimation Soil Transfer (BEST) realizada em laboratório com colunas de acrílico e dois solos distintos, enquanto que as simulações do escoamento superficial foram feitas com Hydrus-1D. Por meio das curvas de infiltração verificou-se que o solo menos arenoso possui uma capacidade de infiltração menor. A primeira simulação mostrou que o escoamento superficial cumulativo foi de 355,67 mm dia-1 enquanto que na segunda simulação o escoamento superficial acumulado foi de 362,03 mm dia-1. A realização dos ensaios de infiltração apenas na superfície do perfil do solo pode acarretar em resultados imprecisos, uma vez que a discrepância entre os valores simulados do escoamento superficial chega a $105,14 \%$. Deste modo, para uma estimativa de escoamento superficial mais precisa são necessários sucessivos ensaios de infiltração em diferentes profundidades.

Palavras-chave: BEST; Hydrus-1D; Parâmetros Hidrodinâmicos; Simulação.

\section{Prediction of runoff and consequent flooding in urban centers}

\begin{abstract}
Flooding is a problem in many urban centers due to the natural de-characterization of the soil and the increase in runoff. The hydrodynamic characterization of soils can be carried out by field or laboratory tests seeking to better predict these floods. The objective of this study was to estimate the surface runoff of water from the soil through simulations using Hydrus-1D, in order to explain the floods that occur in an urban area. To obtain the hydrodynamic parameters, the Beerkan of Estimation Soil Transfer (BEST) test methodology was carried out in a laboratory using acrylic columns and two different soils, while the surface flow simulations were performed using Hydrus-1D. From the infiltration curves, it was found that the less sandy soil has a lower infiltration capacity. The first simulation showed that the cumulative runoff was $355.67 \mathrm{~mm}$ day- 1 while the second simulation found the accumulated runoff to be $362.03 \mathrm{~mm}$ day-1. The performance of infiltration tests on the surface of the soil profile only can lead to inaccurate results, because the discrepancy between the simulated runoff values reaches $105.14 \%$. Thus, for a more accurate runoff estimate, successive infiltration tests at different depths are necessary.
\end{abstract}

Keywords: BEST; Hydrus-1D; Hydrodynamic Parameters; Simulation.

Topic: Ciências do Solo

Reviewed anonymously in the process of blind peer.

Marco Aurélio Calixto Ribeiro de Holanda

Universidade de Pernambuco, Brasil

http://lattes.cnpq.br/2511268845383329

http://orcid.org/0000-0002-0860-2458

holandamacr@yahoo.com.br

Willames de Albuquerque Soares (iD

Universidade de Pernambuco, Brasil

http://lattes.cnpq.br/8129166589399635

http://orcid.org/0000-0003-3268-7241

was@poli.br

Diogo Botelho Correa de Oliveira (it)

Universidade de Pernambuco, Brasil

http://lattes.cnpq.br/2647295852254445

http://orcid.org/0000-0003-1556-5664

dbco_pec@poli.br
Received: 08/08/2020

Approved: $17 / 09 / 2020$
Referencing this:

HOLANDA, M. A. C. R.; SOARES, W. A.; OLIVEIRA, D. B. C.. Predição do escoamento superficial e consequentes alagamentos em centros urbanos. Revista Ibero Americana de Ciências Ambientais, v.11, n.5, p.1-11, 2020. DOI: http://doi.org/10.6008/CBPC21796858.2020.005.0001 


\section{INTRODUÇÃO}

A infiltração pode ser definida como a entrada de água no solo, em seu estado líquido, por meio da interface solo-atmosfera. Entretanto, durante este processo, a capacidade que o solo tem de permitir que a água infiltre tende a diminuir até que o solo sature e os vazios estejam preenchidos majoritariamente por água, (SILVA et al., 2017; OLIVEIRA et al., 2018). Os componentes do balanço de água no solo como a infiltração, a evaporação e o escoamento superficial são dependentes diretos das características físicas do solo, ou seja, da composição granulométrica, do arranjo estrutural das partículas e dos parâmetros hidrodinâmicos (OLIVEIRA et al., 2017).

Atualmente, os alagamentos vem sendo um problema corriqueiro em vários centros urbanos. Tais empecilhos tem sido decorrente da descaracterização natural do solo, a qual gera um aumento no escoamento superficial, a partir da água oriunda da chuva, causando alagamentos (KONRAD, 2014). As localidades mais afetadas geralmente estão próximas de cursos de água, como rios, lagos e represas, uma vez que apresentam cotas mais baixas e passaram por aterramentos e nivelamentos (MIYAZAKI et al., 2012). Estas áreas acabam sofrendo com a pouca profundidade do nível freático, o que deixa a camada superficial mais úmida, aumentando o escoamento superficial. Deste modo, a caracterização físico-hídrica dos solos, mediante a obtenção dos seus parâmetros hidrodinâmicos torna-se essencial para a predição e prevenção de alagamentos em grandes centros urbanos (HOLANDA et al., 2019; VERÇOSA, 2019).

A caracterização da infiltração da água no solo pode ser realizada através de ensaios realizados em campo (Beerkan, infiltrômetro de disco, Beerkan Estimation of Soil Transfer - BEST) ou em laboratório (método inverso, função de pedotransferência e método da evaporação) (SILTECHO et al., 2015). Estes ensaios têm como intuito a estimativa dos parâmetros hidrodinâmicos do solo.

Muitos destes ensaios laboratoriais tem sido realizado através de colunas de solo (EASH et al., 2015). Entretanto, alguns estudos foram e estão sendo desenvolvidos utilizando estes métodos pontuais, apenas nas camadas mais superficiais do solo, e extrapolando os resultados para todo o perfil, supondo que todas as camadas possuem a mesma composição textural e estrutural (WANG et al., 2017). Apesar da composição granulométrica não ser considerada uma informação que sofra grandes variações com o tempo, ela pode ser alterada drasticamente quando ocorrem aterros, por exemplo.

Yang et al. (2004), ao realizarem ensaios em colunas contendo solos arenosos de diferentes composições granulométricas mostraram que há claramente o efeito de uma barreira capilar na interface entre os solos, principalmente entre as camadas de areia média e areia fina do que em solos que contém mais cascalho.

Porém, alguns destes estudos que tem sido conduzido em laboratório, utilizaram tensiômetros e simulações de chuva em colunas de acrílico contendo um único tipo de solo, visando o estudo das características hidrodinâmicas dos solos (LEIJ et al., 2006; IBRAHIM et al., 2014). Contudo Leij et al. (2006) deixa claro que o uso de colunas homogêneas, as quais são compostas por um único tipo de solo, são utilizadas, basicamente, nas modelagens para pequenas profundidades $(0-2000 \mathrm{~mm})$. 
Entretanto, estes métodos (Beerkan, Método da evaporação e infiltrômetro de disco, por exemplo) podem consumir muito tempo e recursos, por isso diversos pesquisadores vem utilizando métodos numéricos para realizar a caraterização hidrodinâmica dos solos (MARTINEZ et al., 2010; ZENG et al., 2018; HOLANDA et al., 2019). Estes métodos, comumente computacionais, visam facilitar este estudo, reduzindo os custos, mas mantendo a precisão e a coerência com a realidade, uma vez que a estimativa de parâmetros como o escoamento superficial depende da acurácia na estimativa da infiltração da água no solo (PAIXÃO et al., 2009; WANG et al., 2017; HOLANDA et al., 2019; HOLANDA et al., 2019).

Nanía et al. (2015) utilizaram modelos hidrológicos e hidráulicos (Storm Water Management Model - SWMM) na previsão de alagamentos em Dalton, Chicago, por meio de parâmetros hidrodinâmicos do solo. Com isso, mostraram que a relação entre a chuva e o escoamento superficial é importante na previsão de alagamentos, uma vez que ela é diretamente responsável pela geração fluxo de água nas ruas.

Mujovo (2014) utilizou modelos computacionais, como o Hydrus-1D, neste tipo de caracterização dos solos de localidades urbanas, afim de realizar um controle da drenagem urbana e consequentemente do balanço hídrico. Sendo assim, objetivou-se estimar o escoamento superficial de água no solo por meio de simulações utilizando o Hydrus-1D, para explicar os constantes alagamentos que ocorrem numa região central da cidade do Recife.

\section{MATERIAIS E MÉTODOS}

A metodologia foi subdividida em três partes: 1) área de estudo, onde é apresentada as características da localidade escolhida, 2) Ensaios de infiltração, onde foi descrito o modo como foram conduzidos estes ensaios e 3) Simulações do fluxo de água no solo, mostrando como o Hydrus-1D foi utilizado.

\section{Área de estudo}

A área de estudo está localizada no bairro da Madalena, o qual encontra-se na parte central da cidade de Recife e é cortado por grandes avenidas que fazem a comunicação entre os bairros da ala leste e oeste da cidade. Boa parte da área demarcada $\left(79.250 \mathrm{~m}^{2}\right), 83 \%$, é composta por solo impermeabilizado e o restante por praças e canteiros. Além disso, uma parte deste bairro é cortada pelo rio Capibaribe, nas proximidades do estádio Adelmar da Costa Carvalho, da Escola Politécnica de Pernambuco (POLI), da Faculdade de Ciências da Administração de Pernambuco (FCAP) e do túnel Chico Science, o qual está localizado há apenas $10 \mathrm{~m}$ de uma de suas margens, Figura 1.

Por conta desta proximidade com corpos hídricos de grande porte, a área de estudo passou por diversos processos de aterro, o que levou a porção superficial do solo a ser composta por materiais com classificação textural de areia, areia franca ou franco-arenoso (OLIVEIRA et al., 2017). Durante os picos de marés altas, que neste estado do país (Pernambuco) possui um regime semi-diurno, as tubulações da rede coletora de águas pluviais que estão direcionadas para o rio ficam parcialmente ou totalmente submersas, dificultando o escoamento. 


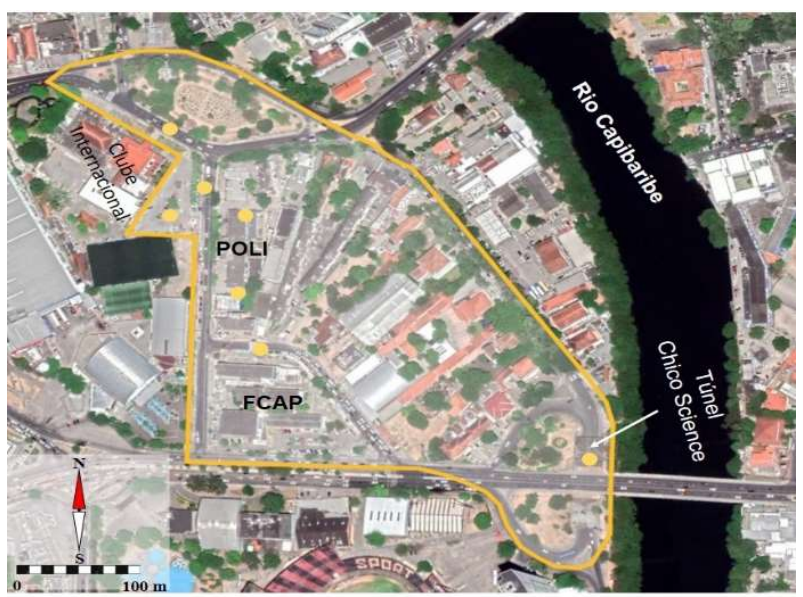

Figura 1: Área de estudo demarcada e pontos de alagamento (círculos amarelos).

Devido a isso, a área de estudo sofre com diversos alagamentos, os quais são intensificados em dias de chuva, principalmente em frente a POLI, ao Clube Internacional do Recife, o qual está situado em frente a uma praça não pavimentada, e dentro do túnel Chico Science. Visando minimizar estes problemas, a rede coletora de drenagem urbana desta localidade tem passado por diversas manutenções, enquanto que o sistema de drenagem do túnel conta com uma bomba de recalque, a qual, dependendo da intensidade do evento chuvoso, não consegue esgotar toda a água com a devida celeridade (MOREIRA, 2014).

Outros centros urbanos e até mesmo turísticos também foram construídos sobre aterros e sofrem com alagamentos, como Miami, na Flórida, a qual é uma cidade costeira que sofre com alagamentos durante os períodos chuvosos. Tais alagamentos também são amplificados durante os picos de maré alta, uma vez que isso acarreta na redução da eficiência da rede de drenagem urbana (WDOWINSKI et al., 2016).

\section{Ensaios de infiltração}

A obtenção dos parâmetros hidrodinâmicos do solo, os quais foram utilizados como dados de entrada do Hydrus-1D, foi feita a partir de ensaios de infiltração realizados em laboratório. Para a montagem, foi utilizada uma coluna de acrílico transparente de $140 \mathrm{~mm}$ de diâmetro com o fundo vazado, a qual foi preenchida com uma camada de drenagem e com solos do tipo franco-arenosos, Figura 2.

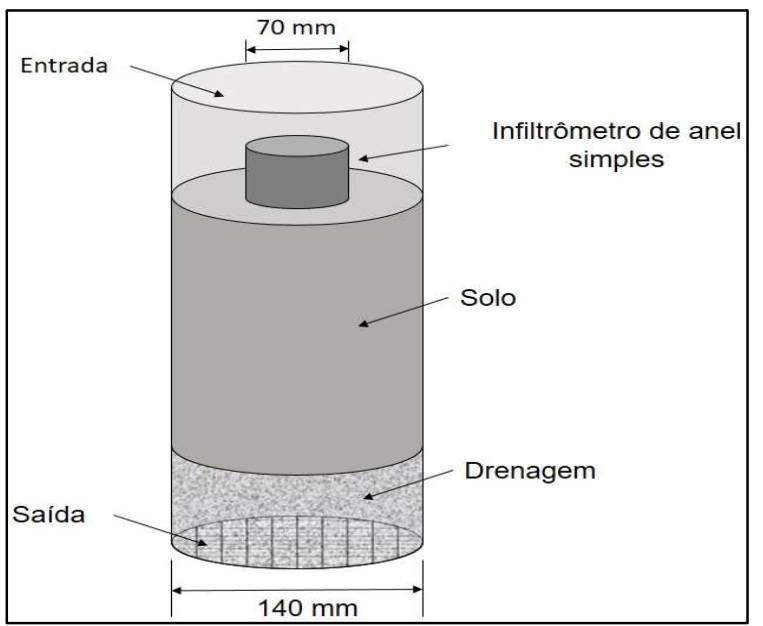

Figura 2: Representação esquemática do aparato experimental.

A realização do ensaio para obtenção dos parâmetros hidrodinâmicos do solo (condutividade 
hidráulica saturada, sorvidade, umidade volumétrica residual e umidade volumétrica saturada) seguiram a metodologia do Beerkan of Estimation Soil Transfer (BEST) proposta por Lassabatère et al. (2006), utilizandose um infiltrômetro de anel simples de $70 \mathrm{~mm}$ de diâmetro e volumes idênticos de água (15 ml). A umidade a umidade volumétrica residual foi considerada igual a zero e a saturada igual a umidade final, enquanto que os tempos de estabilização, $t_{\text {stab}}$, foram obtidos mediante a Equação 1.

$$
t_{s t a b}=\left(\frac{S}{K+\frac{\gamma S^{2}}{r\left(\theta_{f}-\theta_{i}\right)}}\right)
$$

Onde, $S$ representa a sorvidade, $K$ a condutividade hidráulica, $]_{f}$ e $]_{i}$ as umidades volumétricas final e inicial, respectivamente, $r$ o raio do infiltrômetro e $y$ é uma constante que vale 0,75 (SMETTEM et al., 1994).

Durante a montagem de cada coluna, foi separada uma amostra de $150 \mathrm{~g}$ do mesmo solo. Inicialmente, estes $150 \mathrm{~g}$ ficaram expostos às mesmas condições ambientais da coluna, até o momento em que o ensaio de infiltração se iniciou, quando esta amostra foi selada. Após o procedimento experimental, esta amostra de solo foi levada à estufa por $24 \mathrm{~h}$, a $105^{\circ} \mathrm{C}$ e o seu peso, após este período, foi aferido para o cálculo da umidade volumétrica inicial, 国. Após o ensaio de infiltração, outra amostra de solo, de peso conhecido, foi retirada de dentro da coluna e levada à estufa, sendo submetida ao mesmo período de tempo e a mesma temperatura. Após as $24 \mathrm{~h}$, esta segunda amostra foi também pesada para que a umidade volumétrica final, $?_{f}$, pudesse ser determinada.

Foram montados dois ensaios, os quais totalizavam seis colunas de solo. O primeiro deles era composto por três colunas com o mesmo tipo solo, o qual tinha $81,63 \%$ de areia, $3,64 \%$ de silte e $14,73 \%$ de argila. O segundo era composto pelas outras três com um outro solo, o qual era composto por $79,89 \%$ de areia, $5,59 \%$ de silte e $14,52 \%$ de argila. Devido a composição granulométrica, ambos os solos foram classificados como franco-arenosos.

Cada uma das seis colunas continha exatamente a mesma altura de solo, $220 \mathrm{~mm}$, porém as três primeiras, referentes ao primeiro ensaio, continham $4800 \mathrm{~g}$ de solo, e uma densidade aparente aproximada de $1,54 \mathrm{~g} \mathrm{~cm}^{-3}$, enquanto as outras três colunas, referentes ao segundo experimento, foram preenchidas com $5100 \mathrm{~g}$ de solo e uma densidade aparente aproximada de $1,55 \mathrm{~g} \mathrm{~cm}^{-3}$.

\section{Simulações do fluxo de água no solo}

Para a simulação do fluxo de água no solo e do volume de água que escoa sobre o solo (escoamento superficial), foi feito o uso do Hydrus-1D (SIMUNEK et al., 2013), utilizando-se o modelo hidráulico proposto por van Genuchten (1980), Equações 2 e 3. Os perfis dos solos foram montados com uma profundidade de $3000 \mathrm{~mm}$, os quais foram subdivididos de $100 \mathrm{~mm}$ para as condições de contorno. Foram simulados os 30 dias do mês de junho de 2019 (o qual teve uma precipitação acumulada de 463,40 mm), com um passo de tempo de $5 \times 10^{-7}$ dia. Para as condições de fronteira iniciais, superior e inferior, foram consideradas as condições atmosféricas e a drenagem livre, respectivamente. Os parâmetros de entrada dos modelos foram as temperaturas máxima e mínima, a velocidade do vento, a quantidade de horas diárias de sol, a precipitação e a umidade relativa, os quais foram obtidos por meio do banco do Instituto Nacional de 
Meteorologia (INMET) para a estação meteorológica do Curado, que está mais perto do bairro da Madalena.

$$
\begin{gathered}
\theta(h)=\theta_{r}+\frac{\theta_{s}-\theta_{r}}{\left(1+(\alpha h)^{n}\right)^{1-\frac{1}{n}}} \\
K(\theta)=K_{S}\left(\frac{\theta-\theta_{s}}{\theta_{S}-\theta_{r}}\right)^{\frac{1}{2}}\left[1-\left(1-\left(\frac{\theta-\theta_{s}}{\theta_{S}-\theta_{r}}\right)^{\frac{1}{1-\frac{1}{n}}}\right)^{1-\frac{1}{n}}\right]^{2}
\end{gathered}
$$

Onde $\square_{r}$ e 国 representam as umidades volumétricas residual e saturada, $K_{s}$ a condutividade hidráulica saturada, ] e $n$ são os parâmetros de forma e $h$ o potencial matricial.

O perfil da primeira simulação foi montado utilizando-se apenas o solo 1 , o qual é o mais arenoso $(81,63 \%)$, Figura 3a, enquanto que o perfil da segunda simulação foi montado com as proporções de $20 \%$ do solo mais arenoso e $80 \%$ do solo menos arenoso (solo 2). Ou seja, os primeiros $600 \mathrm{~mm}$ do perfil do solo eram compostos pelo solo que continha $81,63 \%$ de areia, enquanto que os $2400 \mathrm{~mm}$ restantes pelo solo que possuía 79,89\% de areia, Figura 3b.

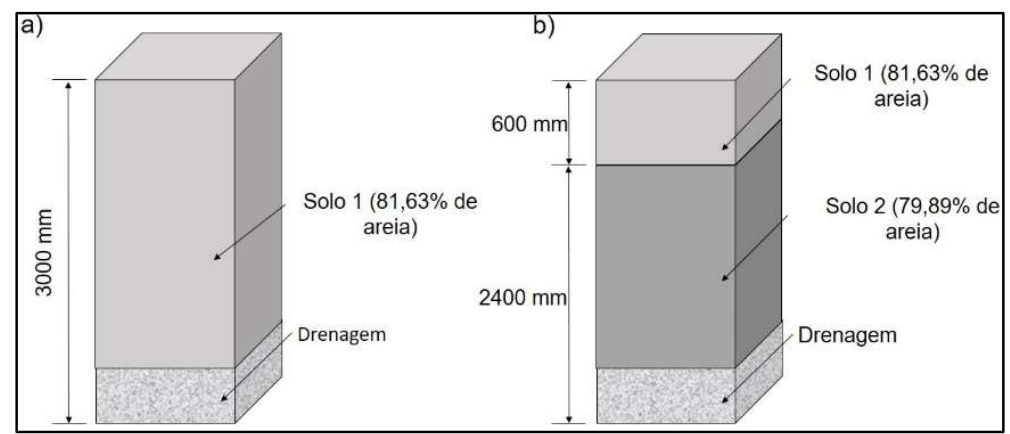

Figura 3: Representação esquemática dos perfis de simulação, a) primeiro perfil e b) segundo perfil.

\section{RESULTADOS E DISCUSSÃO}

Esta secção foi subdividida em duas partes: a primeira contendo os resultados e discussões referentes ao uso do BEST e a segunda contendo os resultados oriundos das simulações realizadas com o Hydrus-1D.

\section{BEST}

Os ensaios de infiltração realizados nas três primeiras colunas, referentes ao primeiro ensaio, duraram em média $673,67 \mathrm{~s}$, enquanto que os ensaios das outras três duraram, em média, 1762,96 s. Utilizando-se ensaios de infiltração foram obtidos os valores da umidade volumétrica saturada ([⿶s), enquanto que a umidade volumétrica residual $\left(\theta_{r}\right)$ foi considerada nula. Por meio do BEST foram obtidas a condutividade hidráulica saturada $\left(K_{s}\right)$ e a sorvidade $(S)$ para os solos de ambos os experimentos. Os valores médios destes respectivos parâmetros hidrodinâmicos dos solos estão presentes na Tabela 1.

Tabela 1: Parâmetros hidrodinâmicos médios obtidos por meio dos ensaios de infiltração.

\begin{tabular}{|l|l|l|l|l|}
\hline Solo & ]$_{r}\left(\mathrm{~cm}^{2} \mathrm{~cm}^{-3}\right)$ & $\mathrm{Z}_{s}\left(\mathrm{~cm}^{-3} \mathrm{~cm}^{-3}\right)$ & $K_{s}\left(\mathrm{~mm} \mathrm{~s}^{-1}\right)$ & $S\left(\mathrm{~mm} \mathrm{~s}^{-0,5}\right)$ \\
\hline 1 & 0 & 0,38 & $4,51 \times 10^{-2}$ & 1,73 \\
\hline 2 & 0 & 0,34 & $1,93 \times 10^{-2}$ & 0,96 \\
\hline
\end{tabular}

A partir destes dados foram montadas as curvas de infiltração e a taxa de infiltração de água no solo para ambos os solos, Figura 4. Estas curvas mostram que o solo 2, que contém 79,89\% de areia, utilizado no 
segundo ensaio possui uma capacidade de infiltração menor, uma vez que para a mesma quantidade de água acumulada o tempo de infiltração foi muito superior ao do primeiro ensaio e o tempo de estabilização também foi maior. Isso se deve ao fato de que os valores de $K_{s}$ e $S$ do segundo experimento são inferiores ao do primeiro, corroborando com os resultados obtidos por Ma et al. (2010), onde eles mostraram que solos de granulometria mais finas conduzem a água com mais dificuldade. O que era de se esperar, uma vez que os solos mais arenosos tendem a conduzirem mais água (DI PRIMA et al., 2018).
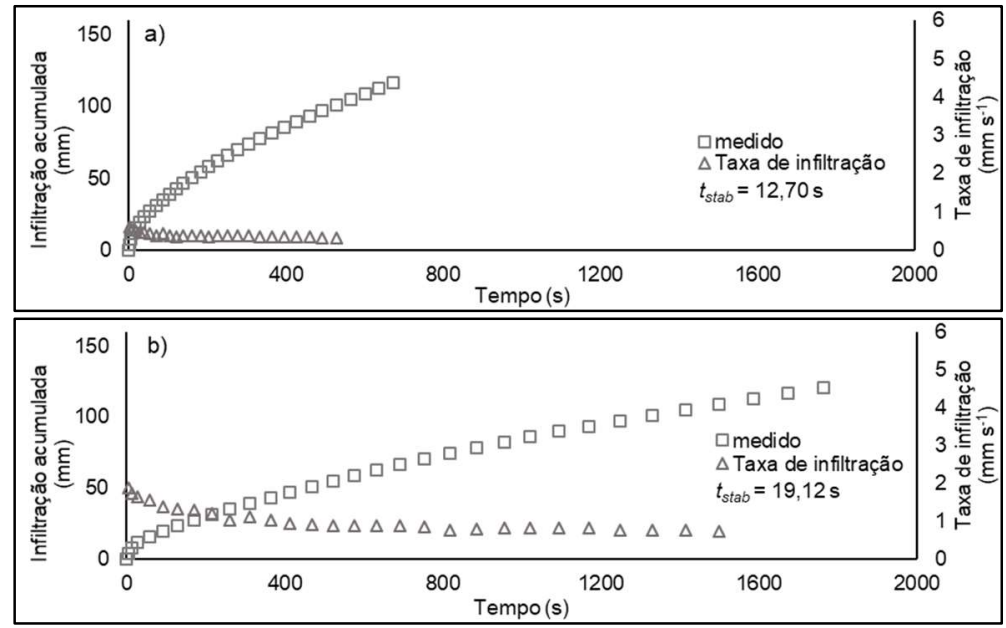

Figura 4: Curvas de infiltração e da taxa de infiltração de água no solo. a) solo 1 e b) solo 2.

\section{Hydrus-1D}

Os resultados da simulação do primeiro perfil, composto apenas pelo solo 1 , realizados com o Hydrus-1D, mostraram que o escoamento superficial cumulativo do período estudado foi de $355,67 \mathrm{~mm} \mathrm{dia}^{-}$ ${ }^{1}$ o que representa, aproximadamente, $76,75 \%$ do total precipitado em todo o período estudado. Além disso, é possível notar que para a maioria dos eventos chuvosos há escoamento superficial, Figura 5, ou seja, o bairro da Madalena está constantemente sofrendo com alagamentos.

Além disso, Soares (2018), ao analisar precipitações de mesma intensidade, porém de diferentes durações, mostrou que para solos do agreste meridional do estado de Pernambuco, os quais são majoritariamente arenosos (até $80,40 \%$ de areia), os eventos de curta duração acarretam em maiores umidades das camadas superficiais do solo. Deste modo, se os eventos chuvosos que ocorreram em junho de 2019 foram rápidos (até 4 horas de duração) então a possibilidade de que tenha sido gerado um escoamento superficial é grande, devido à alta umidade do solo.

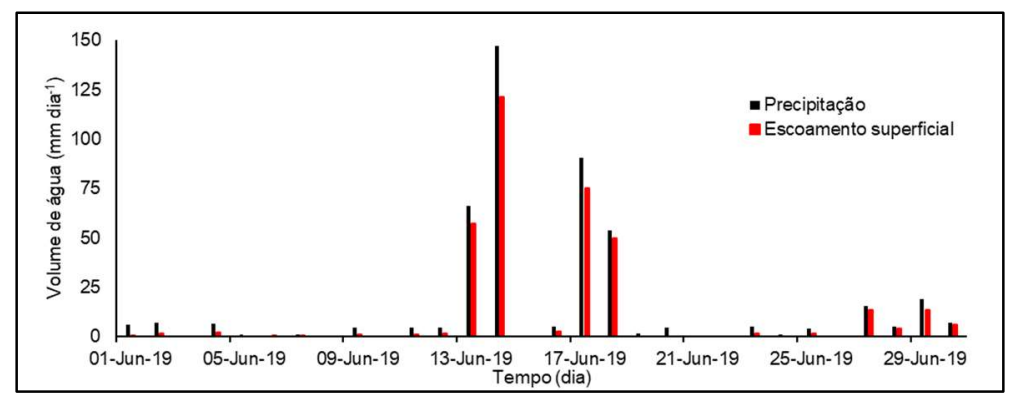

Figura 5: Valores diários de precipitação e de escoamento superficial para o mês de junho/2019 do perfil contendo apenas o solo 1. 
Outro fator que tende a contribuir com os altos valores de escoamento superficial é obsolescência da rede coletora de águas pluviais, a qual foi projetada sem considerar o rápido e massivo crescimento urbano da região. Além disso, o carreamento de sedimentos e resíduos sólidos para dentro da rede pluvial pode gerar acúmulos, reduzindo a secção transversal do conduto e consequentemente, reduzindo a sua capacidade de escoamento dentro da tubulação, aumentando o escoamento superficial e gerando alagamentos (NEVES et al., 2011).

Segundo Wanderley et al. (2018), precipitações acima de $25 \mathrm{~mm}$ dia ${ }^{-1}$ são consideradas fortes, entre 15 e $25 \mathrm{~mm}$ dia$^{-1}$ são moderadas e abaixo de $15 \mathrm{~mm} \mathrm{dia}^{-1}$ são fracas para a cidade de Recife. No dia 23/junho/2019, por exemplo, houve uma chuva fraca, de 5,00 $\mathrm{mm} \mathrm{dia}^{-1}$, que ocasionou um escoamento superficial de $1,76 \mathrm{~mm} \mathrm{dia}^{-1}$, o que representa, um coeficiente de escoamento superficial de aproximadamente 0,35 .

Já no dia 14 de junho de 2019, houve uma chuva de 147,20 mm dia ${ }^{-1}$, a qual gerou um escoamento superficial de $121,48 \mathrm{~mm} \mathrm{dia}^{-1}$, o seja, um coeficiente de escoamento aproximado de 0,82 do total precipitado para este dia. Esse alto valor escoado gerou diversos problemas na cidade do Recife, principalmente na área de estudo, alagando parcialmente o túnel Chico Sience e grandes vias de circulação e totalmente as dependências do estacionamento do Clube Internacional, causando diversos transtornos aos transeuntes, corroborando com o que foi observado por Holanda et al. (2019) ao estudarem os solos não pavimentados da mesma localidade. Além disso, o nível da água dentro das dependências da POLI chegaram a $45 \mathrm{~cm}$ de água, aproximadamente, Figura 6, o que implica em dizer que a rua Benfica, em frente ao campus, encontrava-se alagada com quase $60 \mathrm{~cm}$ de nível de água.

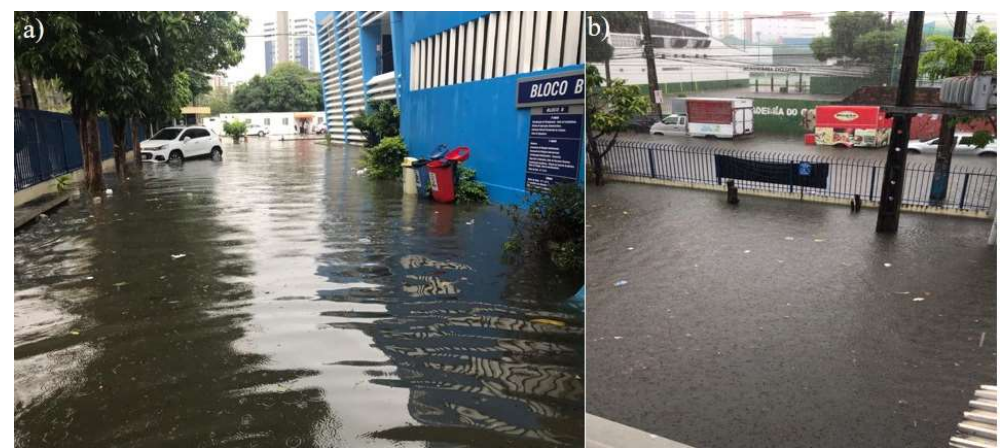

Figura 6: a) Dependências da Escola Politécnica de Pernambuco (POLI) e b) Rua Benfica, em frente a POLI alagadas.

As simulações do segundo perfil, o qual era composto por $20 \%$ do solo 1 e $80 \%$ do solo 2 , mostraram que escoamento superficial acumulado foi de $362,03 \mathrm{~mm} \mathrm{dia}{ }^{-1}$, o que culmina em um coeficiente de escoamento superficial de 0,78 do total precipitado no período estudado. Deste modo, o escoamento superficial desta segunda simulação foi 1,78\% maior que o da primeira, onde só havia um único tipo de solo, ou seja, a mudança da composição granulométrica presente no perfil do solo pode gerar valores de escoamento superficial bem diferentes, mesmo que ambos os solos pertençam a mesma classe textural, ou seja, mesmo que ambos sejam classificados como franco-arenosos.

Estes aumentos também são notados quando se analisa os valores diários do escoamento superficial, Figura 7. Contudo, ainda é muito comum encontrar trabalhos onde foram realizados ensaios de infiltração 
apenas na superfície do solo, e que extrapolam os resultados obtidos, considerando que o perfil é composto pelo mesmo tipo de solo, (SILTECHO et al., 2015; PORZIG et al., 2018; HOLANDA et al., 2019; MORETFERNÁNDEZ et al., 2019).

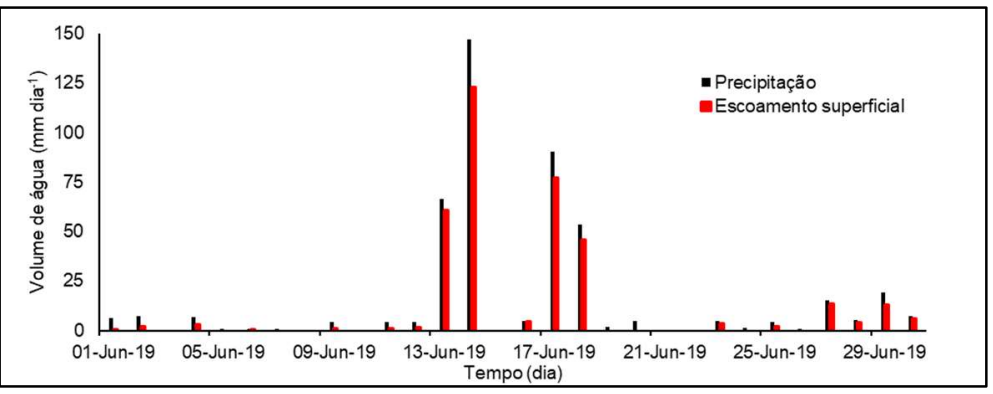

Figura 7: Valores diários de precipitação e de escoamento superficial para o mês de junho/2019 do perfil contendo os solos 1 e 2.

Analisando-se o dia 14 de junho de 2019, nota-se que o escoamento superficial foi 122,84 mm dia-1, o que representa um coeficiente de escoamento aproximado de 0,83 , ou seja, o equivalente a um aumento de $1,12 \%$ em relação ao valor do escoamento, referente ao mesmo dia, obtido na simulação do primeiro perfil. Entretanto, para o dia 23 de junho de 2019 este aumento chega a 105,14\%. Comparando os valores dos escoamentos superficiais de ambas as simulações para o dia 02 de junho de 2019, nota-se que o escoamento superficial obtido com a segunda simulação foi ligeiramente maior que o da primeira, aproximadamente $0,50 \mathrm{~mm} \mathrm{dia}^{-1}$, porém isso representa um aumento de $33,38 \%$ com relação a primeira simulação e um coeficiente de escoamento aproximado de $0,28 \%$ do valor precipitado neste dia. Tais diferenças podem ser observadas na Figura 8.

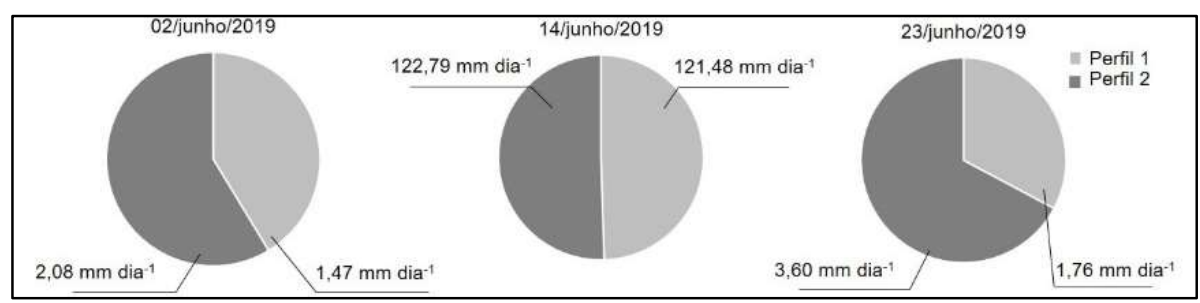

Figura 8: Diferença do escoamento superficial obtidos com as simulações dos dois perfis para os dias 02,14 e 23 de junho de 2019.

\section{CONCLUSÕES}

As simulações dos dois perfis, com um e com dois solos distintos, mostraram que a mudança na composição granulométrica das suas camadas de solo pode acarretar em aumentos significativos, de até $105,14 \%$, no escoamento superficial, mesmo que as camadas sejam compostas por solos de mesma classe textural. Além disso, por meio das simulações realizadas com o Hydrus-1D, também ficou claro que independentemente da composição granulométrica do solo e da intensidade da chuva (fraca, moderada ou forte) houve escoamento superficial na maior parte os dias com chuva.

O comparativo entre os valores do escoamento superficial de ambos os perfis mostrou que a realização de ensaios de infiltração superficiais, com o intuito de caracterizar hidrodinamicamente todo perfil do solo, por meio da premissa de que todas as camadas possuem a mesma composição granulométrica, pode 
acarretar em alagamentos mais frequentes e de maior intensidade. Neste caso, o ideal seria a realização de sucessivos ensaios de infiltração em diferentes profundidades, para que a estimativa do escoamento superficial fosse mais precisa.

\section{REFERÊNCIAS}

DI PRIMA, S.; LASSABATÈRE, L.; ANGULO-JARAMILLHO, R.; PIRASTRU, M.; CERDÀ, A.; KEESSTRA, S.. Laboratory testing of Beerkan infiltration experiments for assessing the role of soil sealing on water infiltration. Catena, v.167, p.373-384, 2018. DOI: http://doi.org/10.1016/j.catena.2018.05.013

EASH, N. S.; SAUER, T. J.; O'DELL, D.; ODOI, E.. Soil Science Simplified. 6 ed. Hoboken: John Wiley \& Sons, 2015.

GENUCHTEN, M. T. Van.. A closed-form equation for predicting the hydraulic conductivity of unsatured soils. Soil Science Society of America Journal, v.44, n.3, p.892-898, 1980. DOI:

http://doi.org/10.2136/sssaj1980.03615995004400050002x

HOLANDA, M. A. C. R.; SOARES, W. A.. Analysis of the effect of impermeability of urban soils on the infiltration of rainwater in the city of Recife, PE. Revista Ambiente e Água, v.14, n.4, p.1-10, 2019. DOI: http://doi.org/10.4136/ambiagua. 2386

HOLANDA, M. A. C. R.; SOARES, W. A.; SILVA, S. R.; OLIVEIRA, D. B. C.. Sensitivity of the Hydrus-1D model to changes in hidrodynamic parameters in yellow latosol. Revista AIDIS de Ingeniéria y Ciencias Ambientales: Investigación, desarrollo y práctica, v.12, n.3, 467-481, 2019. DOI:

http://doi.org/10.22201/iingen.0718378xe.2019.12.3.62969

IBRAHIM, A.; MUKHLISIN, M.; JAAFAR, O.. Rainfall Infiltration through Unsaturated Layered Soil Column. Sains Malaysiana, v.43, n.10, p.1477-1484, 2014.

KONRAD, C. P.. Effects of Urban Development on Floods. Washington: Geological Survey, 2014.

LASSABATÈRE, L.; ANGULO-JARAMILLO, R.; SORIA, J. M.; CUENCA, R.; BRAUD, I.; HAVERKAMP, R.. Beerkan estimation of soiltransfer parameters through infiltration experiments BEST. Soil Science Society of America Journal, v.70, p.521532, 2006. DOI: http://doi.org/10.2136/sssaj2005.0026

LEIJ, F. J.; SCIORTINO, A.; WARRICK, A. W.. Infiltration in two parallel soil columns. Water Resources Research, v.42, p.115, 2006. DOI: http://doi.org/10.1029/2006WR005009

MA, Y.; FENG, S.; SU, D.; GAO, G.; HUO, Z.. Modeling water infiltration in a large layered soil column with a modified Green-Ampt model and HYDRUS-1D. Computers and Electronics in Agriculture, v.71, p.40-47, 2010. DOI: http://doi.org/10.1016/j.compag.2009.07.006

MARTINEZ, M. A.; SILVA, J. B. G.. Modelagem do movimento de sais no solo. In: Manejo de salinidade na agricultura: estudos básicos e aplicados. Fortaleza: INCTsal, 2010. p.93113.

MIYAZAKI, L. C. P.; NUNES, J. O. R.. A relação entre processos morfodinamicos e os desastres naturais: uma leitura das áreas vulneráveis a inundações e alagamentos em Presidente Prudente/SP. Caderno Prudentino de Geografia, v.2, n.34, p.81-96, 2012.

MOREIRA, M. F.. "Tapacurá estourou!": a vulnerabilidade da cidade anfíbia (Recife/PE) aos episódios de inundações e o bairro da Madalena. Tese (Doutorado em Ciências Humanas) - Universidade Federal de Santa Catarinas, Florianópolis, 2014.

MORET-FERNÁNDEZ, D.; LATORRE, B.; GINER, M. L.; RAMOS, J.; ALADOS, C. L.; CASTELLANO, C.; LÓPEZ, M. V.; JIMENEZ, J. J.; PUEYO, Y.. Estimation of the soil hydraulic properties from the transient infiltration curve measured on soils affected by water repellency. Catena, v.178, p.298-306, 2019. DOI: http://doi.org/10.1016/i.catena.2019.03.031

MUJOVO, M. J. N.. Simulação de fluxo vertical em trincheiras de infiltração urbana. Dissertação (Mestrado em Engenharia Ambiental) - Universidade Federal de Santa Catarina, Florianópolis, 2014.

NANÍA, L. S.; LEÓN, A. S.; GARCÍA, M. H.. HydrologicHydraulic Model for Simulating Dual Drainage and Flooding in Urban Areas: Application to a Catchment in the Metropolitan Area of Chicago. Journal of Hydrologic Engineering, v.20, n.5, p.1-13, 2015. DOI: http://doi.org/10.1061/(ASCE)HE.1943-5584.0001080

NEVES, M. G. F. P.; TUCCI, C. E. M.. Composição de resíduos de varrição e resíduos carreados pela rede de drenagem, em uma bacia hidrográfica urbana. Revista de Engenharia Sanitária Ambiental, v.16, p.331-336, 2011. DOI: http://doi.org/10.1590/S1413-41522011000400003

OLIVEIRA, D. B. C.; SOARES, W. A.. Desempenho de modelos de infiltração tridimensional de água no solo. Revista Diálogos, v.18, p.519-544, 2017. DOI: http://doi.org/10.13115/2236-1499v2n18p519

OLIVEIRA, D. B. C.; SOARES, W. A.; HOLANDA, M. A. C. R. Análise de Desempenho de Modelos de Infiltração Unidimensional de Água no Solo. Revista Águas Subterrâneas, v.32, n.1, p.35-42, 2018. DOI: http://doi.org/10.14295/ras.v32i1.28947

PAIXÃO, F. J. R.; ANDRADE, A. R. S.; AZEVEDO, C. A. V.; COSTA, T. L.; GUERRA, H. O. C.. Ajuste de curva de infiltração por meio de diferentes modelos empíricos. Pesquisa Aplicada \& Agrotecnologia, v.2, n.1, p.107-112, 2009.

PORZIG, E. L.; SEAVY, N. E.; OWENS, B. E.; GARDALI, T.. Field Evaluation os a simple infiltration teste and its relationship with bulk density and soil organic carbono in California rangelands. Journal of Soil and Water Conservation, v.73, n.2, p.200-206, 2018. DOI:

http://doi.org/10.2489/jswc.73.2.200 
SILTECHO S.; HAMMECKER C.; SRIBOONLUE V.; CLERMONTDAUPHIN C.; TRELO-GES V.; ANTONINO A. C. D.; ANGULOJARAMILLO R.. Use of field and laboratory methods for estimating unsaturated hydraulic properties under different land uses. Hydrology Earth System Science, v.19, p.11931207, 2015. DOI: http://doi.org/10.5194/hessd-11-6099$\underline{2014}$

SILVA, N. F.; CUNHA, F. N.; FILHO, F. R. C.; MORAIS, W. A.; CUNHA, E. S.; ROQUE, R. C.; ALVES, D. K. M.; TEIXEIRA, M. B.. Métodos para estimative da infiltração de água em um latossolo sob plantio direto e convencional. Global Science Technology, v.10, n.1, p.169-176, 2017.

SIMUNEK, J.; SEJNA, M.; SAITO, H.; SAKAI, M.; VAN GENUCHTEN, M. TH.. The HYDRUS-1D Software Package for Simulating the One-Dimensional Movement of Water, Heat, and Multiple Solutes in Variably-Saturated Media. California: Riverside, 2013.

SMETTEM, K. R. J.; PARLANGE, J. Y.; ROSS, P. J.; HAVERKAMP, R.. Three-dimensional analysis of infiltration from the disc infiltrometer: 1. A cappilary-based theory. Water Resources Research, v.30, p.2925-2929, 1994.

SOARES, W. A.. Análise da dinâmica da água em um solo não saturado sob condições de chuvas simuladas. Revista Águas Subterrâneas, v.32, n.2, p.200-209, 2018. DOI: http://doi.org/10.14295/ras.v32i2.29109

VERÇOSA, L. F. M.. Modelagem hidrológica e hidrodinâmica aplicada à avaliação da ocorrência de inundações na cidade de Recife, PE. Dissertação (Mestrado em Engenharia Civil) Universidade Federal de Pernambuco, Recife, 2019.

WANDERLEY, L. S. A.; NÓBREGA, R. S.; MOREIRA, A. B.; ANJOS, R. S.; ALMEIDA, C. A. P.. As chuvas na cidade do Recife: uma climatologia de extremos. Revista Brasileira de Climatologia, v.22, p.149-164, 2018. DOI: http://doi.org/10.5380/abclima.v22i0.56034

WANG, X.; SAMPLE, D. J.; PEDRAM, S.; SHAO, X.. Performance of two prevalente infiltration models for disturbed urban soils. Hydrology Research, v.48, n.6, p.1520-1536, 2017. DOI: http://doi.org/10.2166/nh.2017.217

WDOWINSKI, S.; BRAY, R.; KIRTMAN, B. P.; WUB, Z.. Increasing flooding hazard in coastal communities due to rising sea level: Case study of Miami Beach, Florida. Ocean \& Coastal Management, v.126, p.1-8, 2016. DOI: http://doi.org/10.1016/j.ocecoaman.2016.03.002

YANG, H.; RAHARDJO, H.; LEONG, E. C.; FREDLUND, D. G.. A study of infiltration on three sand capillary barriers. Canadian Geotechinical Journal, v.41, n.4, 629-643, 2004. DOI: http://doi.org/10.1139/t04-021

ZENG, W.; LEI, G.; ZHA, Y.; FANG, Y.; WU, J.; HUANG, J.. Sensitivity and uncertainty analysis of the HYDRUS-1D model for root water uptake in saline soils. Crop and Pasture Science, v.69, n.2, p.163-173, 2018. DOI: http://doi.org/10.1071/CP17020

A CBPC - Companhia Brasileira de Produção Científica (CNPJ: 11.221.422/0001-03) detém os direitos materiais desta publicação. Os direitos referem-se à publicação do trabalho em qualquer parte do mundo, incluindo os direitos às renovações, expansões e disseminações da contribuição, bem como outros direitos subsidiários. Todos os trabalhos publicados eletronicamente poderão posteriormente ser publicados em coletâneas impressas sob coordenação da Sustenere Publishing, da Companhia Brasileira de Produção Científica e seus parceiros autorizados. Os (as) autores (as) preservam os direitos autorais, mas não tem permissão para a publicação da contribuição em outro meio, impresso ou digital, em português ou em tradução. 